\title{
A Dynamic Intersecting Arrangement Model Based on Isolated Draw Zones for Stope Structure Optimization during Sublevel Caving Mining
}

\author{
Guanghui Li $\mathbb{D}$, ${ }^{1}$ Fengyu Ren $\mathbb{D}^{D},{ }^{1}$ Hangxing Ding $\mathbb{D},{ }^{1}$ Huan Liu $\mathbb{D}^{1},{ }^{1}$ Mingzhi Sun $\mathbb{D}^{1},{ }^{1}$ \\ and Guang $\mathrm{Li} \mathbb{D}^{2}$ \\ ${ }^{1}$ School of Resources and Civil Engineering, Northeastern University, Shenyang 110819, China \\ ${ }^{2}$ Yanqianshan Branch of Ansteel Mining Co., Ltd., Anshan 621010, China \\ Correspondence should be addressed to Hangxing Ding; dinghangxing@mail.neu.edu.cn
}

Received 27 October 2020; Revised 23 November 2020; Accepted 20 December 2020; Published 19 January 2021

Academic Editor: Fateh Mebarek-Oudina

Copyright (C) 2021 Guanghui Li et al. This is an open access article distributed under the Creative Commons Attribution License, which permits unrestricted use, distribution, and reproduction in any medium, provided the original work is properly cited.

\begin{abstract}
In this paper, stope structure optimization during sublevel caving mining is considered under the condition that the isolated draw zones (IDZs) are nonstandard ellipsoid, which is realized by dynamically adjusting the arrangement of IDZs and quantifying the degree of intersection of IDZs according to an ore profit and loss calculation model. A dynamic intersecting arrangement model based on IDZs was proposed, which can dynamically adjust the sublevel height and drift spacing according to the ore-rock bulk flow parameters, economic indicators, occurrence condition of the ore body, drilling machine, and so forth. Based on the model, the range of drift spacing, the lower volume of crestal residual ore, and the higher volume of mixing waste rock are calculated. By deducing the function of ore profit and loss, a calculation model for ore profit and loss is established to quantify the degree of intersection of IDZs and determine the best stope structure. Using the constructed dynamic intersecting arrangement model, a stope structure of $-213 \mathrm{~m}$ to $-303 \mathrm{~m}$ in the Yanqianshan Iron Mine was designed, with a sublevel height of $22.5 \mathrm{~m}$ and a drift spacing of $20.5 \mathrm{~m}$. A physical drawing model was designed, and three physical simulation experiment schemes were conducted to compare and analyse the ore loss and dilution of the intersecting arrangement model and the traditional tangent arrangement model. The results showed that the loss rate decreased by $3.66 \%$ and the dilution rate increased by only $0.22 \%$, thus verifying the effectiveness and applicability of the model to optimize the stope structure.
\end{abstract}

\section{Introduction}

The sublevel caving method is one of the oldest methods for underground mining. This method has many advantages with regard to safety and mechanization, so it is widely used in various kinds of metallic mines worldwide $[1,2]$. With the extension of deep underground mining and the transformation of open-pit mines to underground mines, the application of this mining method is continuously growing, developing towards intelligent mining equipment and stope structure optimization [3]. The stope structure, including sublevel height, drift spacing, and caving spacing, is the main factor that causes ore loss and dilution. An optimal stope structure can adapt to the fluidity of ore-rock, thus reducing ore loss and dilution.
The sublevel caving method is used for mining between sublevels from top to bottom. When ore is extracted, overlying waste rock fills the void created by ore extraction, so the ore is always surrounded by the overlying waste rock. The caved ore is drawn from drifts under loose cover-layerrock, so ore loss and dilution readily occur [4-6]. This is a significant disadvantage of this method. Obviously, the optimal stope structure from a scientific drawing model can accelerate the flow of ore and control the mixing of waste rock, thus mitigating the shortcomings of serious ore loss and dilution $[5,7]$. Therefore, relevant studies that can help improve the recovery and utilization of mineral resources and increase the economic benefit of mines are very significant. 
To optimize the stope structure to reduce ore loss and dilution in sublevel caving mines, scholars have proposed different basic theories; those theories with practical value include ellipsoid draw theory and stochastic medium draw theory $[8,9]$. These theories study the movement trends of caved ore and overlying waste rock but adopt different research methods [10]. Ellipsoid draw theory experimentally proves that isolated draw zones (IDZs) (the geometrical locus defined by the initial location of the caved ore that is recovered from a drawpoint in underground mining) are approximately ellipsoids [11]. Then, the properties of IDZs are studied and used to explain the flow characteristics of the ore-rock bulk. Stochastic medium draw theory simplifies the ore-rock bulk into a continuous flowing random medium and then studies the movement process of the ore-rock bulk through probability theory $[12,13]$. In the final analysis, these theories are ultimately used to optimize caving mining schemes and to determine reasonable stope structures. Based on ellipsoid draw theory, Kvapil [14] expanded from a single IDZ and used IDZ spatial arrangement theory to design stope structures. This design principle later evolved into two stope structures: wide drift spacing structure and high sublevel structure. In wide drift spacing structure, the upper and lower ellipsoids are directly tangent, while the left and right discharge ellipsoids are separated and tangent to the upper and lower ellipsoids (see Figure 1(a)). Similarly, in high sublevel structure, the left and right ellipsoids are directly tangent to each other, while the upper and lower discharge ellipsoids are separated and tangent to both the left and right ellipsoids (see Figure 1(b)). According to the design principle, the sublevel height of caving mines worldwide is $15 \mathrm{~m}$ to $30 \mathrm{~m}$, and the drift spacing is generally slightly less than or equal to the sublevel height. However, under these stope structures, a certain proportion of ore is outside the IDZ (see Figure 1); therefore, this cannot be regarded as the optimal stope structure, and it has been proven that these stope structures do not yield a good recovery effect in practical mining applications. Some mines, such as the Xiadian Gold Mine in China and the Kamoto Copper Mine in Zambia, have recoveries of less than $80 \%$ [15]. Yuan et al. [16] analysed the coincident relationship between the caved ore, crestal residual ore, and IDZ and concluded that, within a certain temporal period and spatial area, increasing the distance between the drawpoints reduces the recovery rate of ore. Wang et al. [17] numerically simulated drift spacings of $12 \mathrm{~m}$ to $20 \mathrm{~m}$, and the results showed that there were no obvious changes in the waste in-ore rate as the drift spacing increased, but the ore recovery rate decreased from $63 \%$ to $46 \%$ with increasing drift spacing. Tan et al. [18] discussed the wide drift spacing structure based on the ellipsoid drawing theory and pointed out the defects of the design scheme. The above studies are all based on qualitative descriptions of stope structure and analyse the influence of drift spacing on ore recovery. The specific stope structure is still determined according to mining experience and experiments. Moreover, for the whole mine, the stope structure is fixed and cannot be dynamically adjusted.

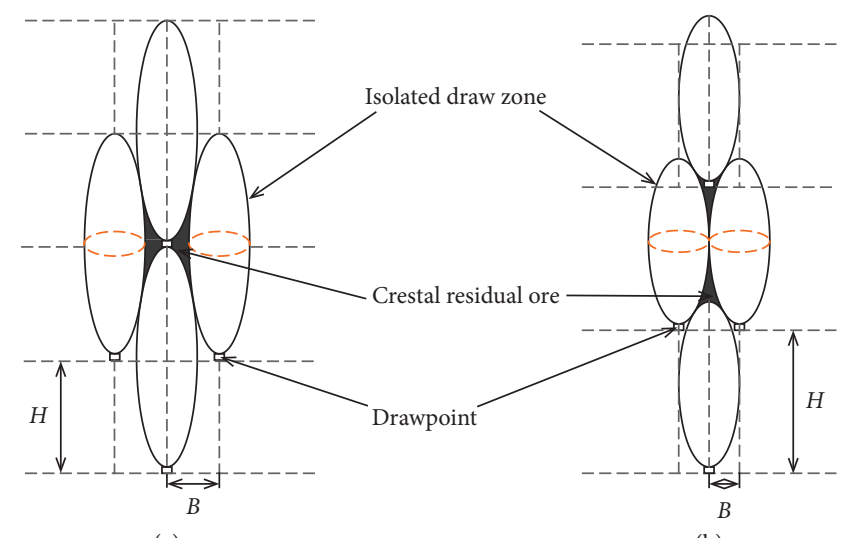

(a)

(b)

FIgURE 1: The conventional tangent arrangement model based on IDZs [14]. (a) Wide drift spacing structure. (b) High sublevel structure.

When mining by the sublevel caving method, the shape of the IDZ is affected by many factors, including not only the physical properties of particles, such as the ore-rock bulk geometry, lumpiness, concrete gradation, humidity, and loose factor, but also the mechanical properties of particles, such as the lateral pressure and friction [19]. Therefore, the shape of the IDZ is not a regular ellipsoid. Marano et al. $[9,20]$ believe that the IDZ is composed of different shapes, such as an ellipsoid in the upper part and a parabolic rotating body in the lower part. Janelid and Kvapil [3, 21, 22] proved that the ellipsoid is an approximate ellipsoid, but there are cases in which the upper part is thick and the lower half is thin and vice versa. The nonstandard ellipsoid shape of the IDZ means that the tangent arrangement model cannot be completely applied to the design of the stope structure.

In this study, stope structure optimization during sublevel caving mining is considered under the condition that the IDZs are nonstandard ellipsoids, which is realized by dynamically adjusting the arrangement of IDZs and quantifying the degree of intersection of IDZs according to an ore profit and loss calculation model. A dynamic intersecting arrangement model based on IDZs which can dynamically adjust the stope structure according to the ore-rock bulk flow parameters, economic indicators, occurrence conditions of ore bodies, drilling machines, and so forth is proposed. Then, taking the ore profit and loss as the research object, a calculation model is established to quantify the degree of intersection of the IDZs and determine the best sublevel height and drift spacing. Finally, with the dynamic intersecting arrangement model, the optimal stope structure of the Yanqianshan Iron Mine is designed; moreover, three experimental schemes using the Yanqianshan Iron Mine as the background were carried out to compare the tangent arrangement model based on IDZs and the intersecting arrangement model based on IDZs.

\section{The Intersecting Arrangement Model Based on IDZs}

The design and optimization of the stope structure generally follow the principle that the shape of the caved ore heap is 
consistent with that of the IDZ [14], which means that the IDZ should contain as much caved ore and residual ore as possible. According to the conclusion of Yuan and Liu $[16,23]$, if the distance between drawpoints is reduced so that the IDZs intersect, the crestal residual ore will be reduced (see Figure 2), which has a significant impact on the ore recovery rate. Therefore, the intersecting arrangement model based on IDZs can better reflect the principle that the shape of the caved ore heap is consistent with that of the IDZ, especially when the IDZ is a nonstandard ellipsoid.

Stochastic medium draw theory of Ren [12] simplifies the ore-rock bulk into a continuous flowing random medium, which better reflects the shape of the IDZ. Based on stochastic medium draw theory, for the end ore drawing of the sublevel caving method, the shape of IDZ is given by

$$
\frac{\left(x-k z^{(\alpha / 2)}\right)^{2}}{\beta z^{\alpha}}+\frac{y^{2}}{\beta_{1} z^{\alpha_{1}}}=\left(\frac{\alpha+\alpha_{1}}{2}+1\right) \ln \frac{2 H}{z} \text { [12], }
$$

where $\alpha, \beta, \alpha_{1}$, and $\beta_{1}$ are the ore-rock bulk flow parameters ( $\alpha$ and $\beta$ represent the vertical direction of the drift, and $\alpha_{1}$ and $\beta_{1}$ represent the horizontal direction of the drift); $k$ is the impact coefficient of the ore body and depends on the damping degree of the ore-rock bulk by the ore body (in general, $k=0.1 \sim 0.15$ ); and $H$ is the sublevel height. Equation (1) is mainly used to determine the width of the IDZ in the vertical direction of the drift to design the drift spacing.

When mining by the sublevel caving method, the shape of the IDZ is not a regular ellipsoid, and the tangent arrangement model based on IDZs will reduce the density of the spatial arrangement, causing more ore to be outside the range of the IDZ. Under these conditions, it is more appropriate to adopt the intersecting arrangement model based on IDZs. The drift spacing is shortened so that IDZs $R_{2}$ and $R_{3}$ intersect with the upper and lower IDZs $R_{1}$ and $R_{3}$, respectively, and then the crestal residual ore will be reduced (see Figure 3). Of course, this shortening will promote the mixing of waste rock, but this problem can be addressed by calculating the comprehensive economic benefits of the ore. The best intersection degree of the IDZ corresponds to the maximum economic benefit.

\section{Optimization of the Stope Structure}

The sublevel height is determined by occurrence conditions of the ore body, drilling machine, demolition equipment, and so forth. Once the sublevel height is determined, it is difficult to change. The drift spacing is a quantitative representation of the intersecting arrangement model based on IDZs, and the degree of intersection determines the drift spacing. In this section, on the basis of establishing the intersecting arrangement model based on IDZs, the method for determining the optimal stope structure is given.

3.1. Range of the Drift Spacing. In this section, equations of the four adjacent IDZs are established at the same coordinate according to the shape of the IDZ (equation (1)). On this basis, we derived the calculation method of the width of the IDZ in the vertical direction of the drift and provided the

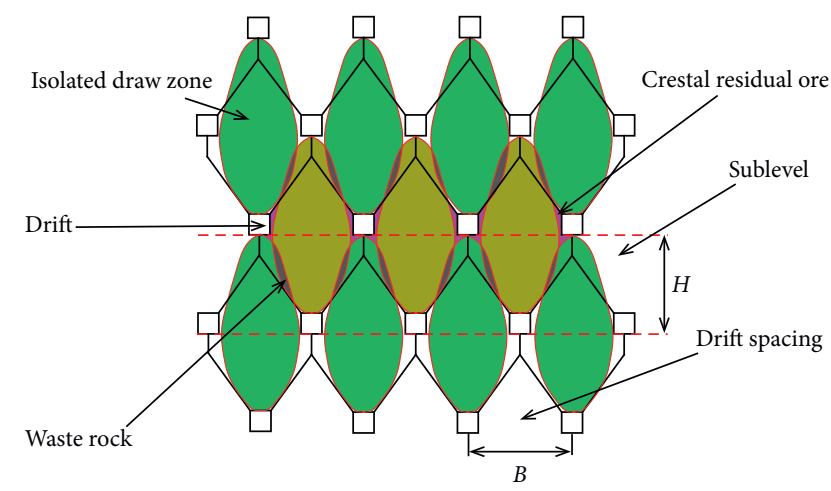

FIGURE 2: Stope structure of the intersecting arrangement model based on IDZs.

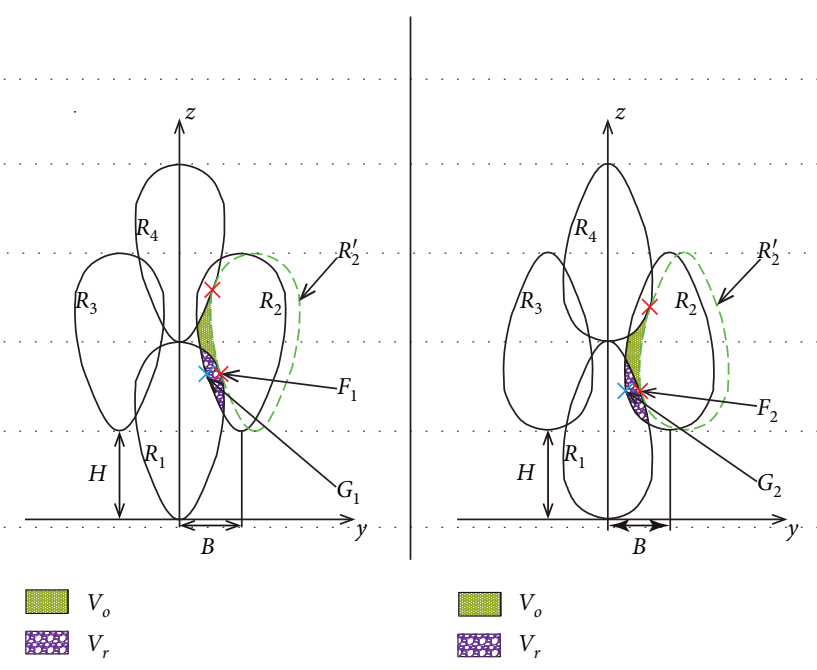

(a)

(b)

FIGURE 3: The intersecting arrangement model based on IDZs. (a) $\alpha$ $<1.44$. (b) $\alpha>1.44$.

determination method for the drift spacing by using the arrangement of the IDZs.

From equation (1) and Figure 3, the equation of the IDZ $R_{1}$ is as follows:

$$
R_{1}: \frac{\left(x-k z^{(\alpha / 2)}\right)^{2}}{\beta z^{\alpha}}+\frac{y^{2}}{\beta_{1} z^{\alpha_{1}}}=\left(\frac{\alpha+\alpha_{1}}{2}+1\right) \ln \frac{2 H}{z} \text { [12]. }
$$

By transforming the coordinates of the centre point of IDZ $R_{1}$, the equations of $R_{2}, R_{3}$, and $R_{4}$ are as follows:

$$
R_{2}: \frac{\left(x-k(z-H)^{(\alpha / 2)}\right)^{2}}{\beta(z-H)^{\alpha}}+\frac{(y-B)^{2}}{\beta_{1}(z-H)^{\alpha_{1}}}=\left(\frac{\alpha+\alpha_{1}}{2}+1\right) \ln \frac{2 H}{z-H},
$$

$$
R_{3}: \frac{\left(x-k(z-2 H)^{(\alpha / 2)}\right)^{2}}{\beta(z-2 H)^{\alpha}}+\frac{y^{2}}{\beta_{1}(z-2 H)^{\alpha_{1}}}=\left(\frac{\alpha+\alpha_{1}}{2}+1\right) \ln \frac{2 H}{z-2 H},
$$




$$
R_{4}: \frac{\left(x-k(z-H)^{(\alpha / 2)}\right)^{2}}{\beta(z-H)^{\alpha}}+\frac{(y+B)^{2}}{\beta_{1}(z-H)^{\alpha_{1}}}=\left(\frac{\alpha+\alpha_{1}}{2}+1\right) \ln \frac{2 H}{z-H},
$$

where $B$ is the drift spacing, which refers to the distance between two drawpoints; $R_{1}$ is the lower IDZ; $R_{2}$ is the right IDZ; $R_{3}$ is the upper IDZ; and $R_{4}$ is the left IDZ.

For $R_{1}$, along the direction of $x=k z \hat{(\alpha / 2)}$, which is the vertical direction of the drift, the profile equation of $R_{1}$ is as follows:

$$
y^{2}=\left(\frac{\alpha+\alpha_{1}}{2}+1\right) \beta_{1} z^{\alpha_{1}} \ln \frac{2 H}{z}
$$

For $R_{2}$, along the direction of $\left.x=k(z-H) \hat{(} \alpha / 2\right)$, which is the vertical direction of the drift, the profile equation of $R_{2}$ is as follows:

$$
(y-B)^{2}=\left(\frac{\alpha+\alpha_{1}}{2}+1\right) \beta_{1}(z-H)^{\alpha_{1}} \ln \frac{2 H}{(z-H)} .
$$

The height of the widest part of $R_{1}$ is denoted by $h$, and the normal slope of $R_{1}$ at $z=h$ is zero; for equation (6), $\mathrm{d} y / \mathrm{d} z=0$. Then,

$$
z=h=2 H e^{-\left(1 / \alpha_{1}\right)} .
$$

Next, the maximum and minimum values of the drift spacing are calculated for different IDZ shapes.

(1) $\alpha<1.44$

The widest part of the IDZ is located at the lower part, and the IDZ is similar to an ellipsoid with a thin upper part and thick lower part (see Figure 3(a)).

For $R_{2}$, by equations (7) and (8), the widest part is located at $z$, where

$$
z=2 H e^{-\left(1 / \alpha_{1}\right)}+H .
$$

Point $G_{1}$ is defined as follows:

$$
G_{1}\left(2 H e^{-\left(1 / \alpha_{1}\right)}+H,-\sqrt{\frac{\left(\left(\alpha+\alpha_{1} / 2\right)+1\right) \beta_{1}(2 H)^{\alpha_{1}}}{\alpha_{1} e}}+B\right) \text {. }
$$

For $R_{1}$, by equations (6), (8), and (9), point $F_{1}$ is defined as follows:

$$
F_{1}\left(2 H e^{-\left(1 / \alpha_{1}\right)}+H, \sqrt{\left(\frac{\alpha+\alpha_{1}}{2}+1\right) \beta_{1} H^{\alpha_{1}}\left(1+2 e^{-\left(1 / \alpha_{1}\right)}\right)^{\alpha_{1}} \ln \frac{2}{1+2 e^{-\left(1 / \alpha_{1}\right)}}}\right)
$$

When $R_{2}$ is tangent to $R_{1}$ and $R_{3}$, the drift spacing is maximized, and points $G_{1}$ and $F_{1}$ coincide; therefore,

$$
B \max =\sqrt{\left(\frac{\alpha+\alpha_{1}}{2}+1\right) \beta_{1} H^{\alpha_{1}}\left(1+2 e^{-\left(1 / \alpha_{1}\right)}\right)^{\alpha_{1}} \ln \frac{2}{1+2 e^{-\left(1 / \alpha_{1}\right)}}}+\sqrt{\frac{\left(\left(\alpha+\alpha_{1} / 2\right)+1\right) \beta_{1}(2 H)^{\alpha_{1}}}{\alpha_{1} e}} .
$$

When $R_{2}$ is tangent to the $z$-axis, the drift spacing is minimized; therefore,

$$
B \min =\sqrt{\frac{\left(\left(\alpha+\alpha_{1} / 2\right)+1\right) \beta_{1}(2 H)^{\alpha_{1}}}{\alpha_{1} e}}
$$

where $B_{\max }$ is the maximum drift spacing and $B_{\min }$ is the minimum drift spacing.

(2) $\alpha>1.44$
The widest part of the IDZ is located in the upper part, and the IDZ is similar to an ellipsoid with a thick upper part and thin lower part (see Figure 3(b)).

For $R_{1}$, according to equations (6) and (8), the widest part is located at $z$, and point $F_{2}$ is defined as follows:

$$
F_{2}\left(2 H e^{-\left(1 / \alpha_{1}\right)}, \sqrt{\frac{\left(\left(\alpha+\alpha_{1} / 2\right)+1\right) \beta_{1}(2 H)^{\alpha_{1}}}{\alpha_{1} e}}\right) .
$$

For $R_{2}$, according to equations (7) and (8), point $G_{2}$ is defined as follows:

$$
G_{2}\left(2 H e^{-\left(1 / \alpha_{1}\right)},-\sqrt{\left(\frac{\alpha+\alpha_{1}}{2}+1\right) \beta_{1} H^{\alpha_{1}}\left(2 e^{-\left(1 / \alpha_{1}\right)}-1\right)^{\alpha_{1}} \ln \frac{2}{2 e^{-\left(1 / \alpha_{1}\right)}-1}}+B\right)
$$


When $R_{2}$ is tangent to $R_{1}$ and $R_{3}$, the drift spacing is maximized, and points $G_{2}$ and $F_{2}$ coincide:

$$
B \max =\sqrt{\left(\frac{\alpha+\alpha_{1}}{2}+1\right) \beta_{1} H^{\alpha_{1}}\left(2 e^{-\left(1 / \alpha_{1}\right)}-1\right)^{\alpha_{1}} \ln \frac{2}{2 e^{-\left(1 / \alpha_{1}\right)}-1}}+\sqrt{\frac{\left(\left(\alpha+\alpha_{1} / 2\right)+1\right) \beta_{1}(2 H)^{\alpha_{1}}}{\alpha_{1} e}} .
$$

When $R_{2}$ is tangent to the $z$-axis, the drift spacing is minimized:

$$
B \min =\sqrt{\frac{\left(\left(\alpha+\alpha_{1} / 2\right)+1\right) \beta_{1}(2 H)^{\alpha_{1}}}{\alpha_{1} e}}
$$

In summary, when the IDZ is a nonstandard ellipsoid, the range of the drift spacing is as follows:

$$
\left[\begin{array}{l}
\sqrt{\frac{\left(\left(\alpha+\alpha_{1} / 2\right)+1\right) \beta_{1}(2 H)^{\alpha_{1}}}{\alpha_{1} e}} \\
\sqrt{\left(\frac{\alpha+\alpha_{1}}{2}+1\right) \beta_{1} H^{\alpha_{1}}\left(1+2 e^{-\left(1 / \alpha_{1}\right)}\right)^{\alpha_{1}} \ln \frac{2}{1+2 e^{-\left(1 / \alpha_{1}\right)}}}
\end{array}\right.
$$$$
\left.+\sqrt{\frac{\left(\left(\alpha+\alpha_{1} / 2\right)+1\right) \beta_{1}(2 H)^{\alpha_{1}}}{\alpha_{1} e}}\right] \quad \alpha<1.44,
$$$$
\left[\begin{array}{l}
\sqrt{\frac{\left(\left(\alpha+\alpha_{1} / 2\right)+1\right) \beta_{1}(2 H)^{\alpha_{1}}}{\alpha_{1} e}} \\
\sqrt{\left(\frac{\alpha+\alpha_{1}}{2}+1\right) \beta_{1} H^{\alpha_{1}}\left(2 e^{-\left(1 / \alpha_{1}\right)}-1\right)^{\alpha_{1}} \ln \frac{2}{2 e^{-\left(1 / \alpha_{1}\right)}-1}}
\end{array}\right.
$$$$
\left.+\sqrt{\frac{\left(\left(\alpha+\alpha_{1} / 2\right)+1\right) \beta_{1}(2 H)^{\alpha_{1}}}{\alpha_{1} e}}\right] \quad \alpha>1.44 .
$$

3.2. Volume Calculation. When the sublevel height is determined, with decreasing drift spacing, the arrangement model based on IDZs changes from tangent to intersecting, the amount of crestal residual ore decreases, and the mixing amount of waste rock increases. $V_{o}$ is the lower crestal residual ore volume, and $V_{r}$ is the higher mixing waste rock volume. When $V_{o}$ and $V_{r}$ are calculated, the functional relations of $V_{o}$ and $B$ as well as those of $V_{r}$ and $B$ are fitted (see Figure 4).

The solution steps are as follows:

Step 1: determine the sublevel height, parameter value, and range of $B$. The sublevel height is determined by occurrence conditions of the ore body, drilling machine, demolition equipment, and so forth. Once the sublevel height is determined, it is difficult to change. The parameters include the ore-rock bulk flow parameters $\left(\alpha, \beta, \alpha_{1}\right.$ and $\left.\beta_{1}\right)$, the impact coefficient of the ore body $(k)$, and the sublevel height $(H)$. The ore-rock bulk flow parameters can be obtained by laboratory or mining field drawing experiments, and the impact coefficient of the ore body is generally between 0.1 and 0.15 . The range of $B$ can be determined by equation (18) or (19).

Step 2: divide the areas of $V_{o}$ and $V_{r}$. As shown in Figure 3, the areas of $V_{o}$ and $V_{r}$ can be calculated by $V_{o}=1 / 2\left[R_{2}-\left(R_{2} \cap R_{2}^{\prime}\right)-\left(R_{2} \cap R_{1}\right)-\left(R_{2} \cap R_{4}\right)\right]$ and $V_{r}=R_{1} \cap R_{2}$.

Step 3: find the Monte Carlo solution for $V_{o}$ and $V_{r}$. With equations (2)-(5) and the parameters that are determined in step 1, the IDZ boundary is established. Using the Monte Carlo method with the IDZ boundary, the membership function, which is used to judge whether a point is within the boundary of the IDZ, is written. Statistical points in the $V_{o}$ and $V_{r}$ areas and $V_{o}$ and $V_{r}$ are determined [24, 25].

Step 4: determine the functional relationship between $V_{o}$ and $B$ as well as that between $V_{r}$ and $B$. Data analysis is performed on $V_{o}$ and $B$ as well as $V_{r}$ and $B$, and then the functional relationship between $V_{o}$ and $B$ and that between $V_{r}$ and $B$ are fitted.

$$
\begin{aligned}
& V o=f_{1}(B), \\
& V r=f_{2}(B) .
\end{aligned}
$$

3.3. Economic Evaluation. The direct purpose of mining enterprises is to exploit ore, and the final profit product is the ore concentrate. To determine the optimal intersecting arrangement model based on IDZs, the economic benefit of the concentrate is taken as the final evaluation target. First, the calculation formula for ore profit and the calculation formula for ore loss are established. Then, combined with 


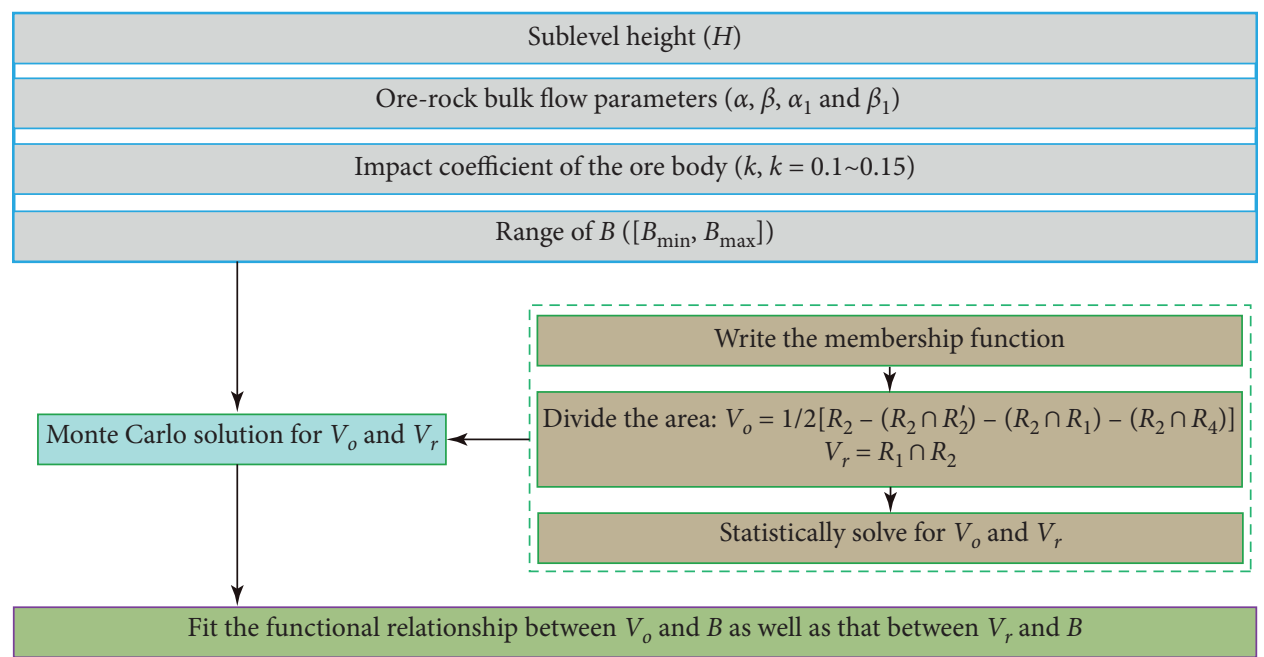

FIgURE 4: Technical route of the volume calculation.

equation (20), the ore profit and loss are calculated. Finally, the ore profit and loss are analysed comprehensively, and the optimal solution is determined to obtain the best stope structure.

3.3.1. Ore Profit and Loss. (1) Ore Profit. The crestal residual ore from drawing to dressing eventually becomes concentrate; in this process, the profit mainly considers the concentrate profit and cost loss [26].

The concentrate profit refers to the value of the concentrate produced after the ore is processed by the beneficiation plant [27].

The ore is processed by the beneficiation plant and finally becomes concentrate and tailings. According to the conservation of quality, one ton of ore is processed into concentrate and tailings, so $1=Q_{c}+Q_{t}$. The conservation of the metal content is

$$
\begin{aligned}
1 \times c_{o} & =Q_{c} \times c_{c}+Q_{t} \times c_{t}[27], \\
Q_{c} & =\frac{c_{o}-c_{t}}{c_{c}-c_{t}} .
\end{aligned}
$$
is

The profit from converting a ton of ore into concentrate

$$
p_{1}=Q_{c}=\frac{\left(c_{o}-c_{t}\right)}{\left(c_{c}-c_{t}\right)} \times P
$$

where $Q_{c}$ is the quality of the concentrate, $Q_{t}$ is the quality of tailings, $C_{o}$ is the ore grade, $C_{c}$ is the concentrate grade, $C_{t}$ is the tailings grade, $p_{1}$ is the profit of converting a ton of ore into concentrate, and $P$ is the concentrate price.

The cost loss refers to the costs incurred by ore from drawing to dressing due to upgrading, ore blending, transportation, and so forth. Mining enterprises can statistically determine the actual technical and economic indicators of the year to obtain the cost loss per ton of ore, which is represented by $p_{2}$ [28].
Based on the above analysis, the profit generated by recovering one ton of crestal residual ore is $p_{1}-p_{2}$, and, with equation (23),

$$
p=\frac{\left(c_{o}-c_{t}\right)}{\left(c_{c}-c_{t}\right)} \times P-p_{2},
$$

where $p$ is the ore profit.

(2) Ore Loss. The mixing of waste rock results in ore dilution, and the loss caused by this dilution mainly considers the loss of metal from tailings and the cost of waste rock mining and processing [26].

The loss of metal from tailings refers to waste rock that is of no grade or is lower than tailings grade; when this rock is treated as tailings, it needs to be matched with some lowgrade ore, causing metal loss [27].

For one ton of waste rock in tailings, the resulting metal loss is

$$
Q_{m}=\frac{1}{1-c_{t}} \times c_{t}[27]
$$

where $Q_{m}$ is the metal quality.

The amount of metal lost due to one ton of waste rock in tailings is expressed as the amount of concentrate:

$$
Q_{m}=Q_{c} \times c_{c} .
$$

The conservation of the metal content is

$$
\frac{1}{1-c_{t}} \times c_{t}=Q_{c} \times c_{c} .
$$

The loss of metal from converting a ton of waste rock into tailings is converted into concentrate:

$$
Q_{c}=\frac{c_{t}}{c_{c} \times\left(1-c_{t}\right)} \text {. }
$$

The loss of metal from tailings is

$$
l_{1}=\frac{c_{w}}{c_{j} \times\left(1-c_{w}\right)} \times P,
$$


where $l_{1}$ is the loss of metal from tailings.

The cost of waste rock mining and processing refers to the expenses associated with mining, processing, transportation and stacking, and so forth incurred by the mixture of waste rock. Mining enterprises can calculate the actual technical and economic indicators of the year to obtain the cost loss per ton of waste rock, which is represented by $l_{2}$.

Based on the above analysis, the loss generated by mixing one ton of waste rock is $l_{1}+l_{2}$, and, with equation (29),

$$
l=\frac{c_{w}}{c_{j} \times\left(1-c_{w}\right)} \times P+l_{2},
$$

where $l$ is the ore loss.

3.3.2. Optimal Path Spacing. When the spatial arrangement model based on IDZs changes from a tangent arrangement to an intersecting arrangement, as the drift spacing decreases, the amount of crestal residual ore decreases but the amount of mixed waste rock increases. A smaller amount of crestal residual ore increases the profitability of the mine, and a larger amount of mixing waste rock increases the mine loss. With equations (24) and (30), the economy of the intersecting arrangement model based on IDZs can be calculated as

$$
s=V_{O} \times \rho_{O} \times p-V_{r} \times \rho_{r} \times l,
$$

where $\rho_{o}$ is the ore density and $\rho_{r}$ is the rock density.

By equations (20), (31) can be rewritten as follows:

$$
s=f_{1}(B) \times \rho_{o} \times p-f_{2}(B) \times \rho_{r} \times l .
$$

Differentiating equation (32) with respect to $B$ yields the drift spacing with the highest benefit, that is, the optimal intersection degree of IDZs.

\section{Application of the Model}

4.1. Engineering Background. The Yanqianshan Iron Mine is located in the Anshan mining area in Northeast China, the largest mining area currently in China. After the previous open-pit mining phase, there were approximately 357.27 million tons of geological reserves outside the open-pit boundary. In 2009, the mine officially entered the transition period from open pit to underground mining, which lasted for 5 years. After the transition period, the mine was fully converted to underground mining and the sublevel caving method was adopted (see Figure 5). The stope structure parameters are as follows: the sublevel height is $18 \mathrm{~m}$ and the drift spacing is $20 \mathrm{~m}$. Under the conditions of the stope structure, the loss rate and dilution rate are approximately $15 \%$ and $18 \%$, respectively, and there is still room for improvement [29, 30].

In 2019, the design of the mining scheme for elevations of $-213 \mathrm{~m}$ to $-303 \mathrm{~m}$ in the Yanqianshan Iron Mine began in preparation for production over the next five years. Under the premise that the mining equipment meets the production needs, the intent is to divide the ore body from elevations of $-213 \mathrm{~m}$ to $-303 \mathrm{~m}$ into four sublevels; then, the sublevel height is $22.5 \mathrm{~m}$. This can reduce the cost of preliminary mining and the cutting of a sublevel (see Figure 6). However, the drift spacing has not been matched with the sublevel height, and the drift spacing has been included in the design and experimentation of the Yanqianshan Iron Mine.

According to the intersecting arrangement model based on IDZs, the optimal stope structure of elevations from $-213 \mathrm{~m}$ to $-303 \mathrm{~m}$ in the Yanqianshan Iron Mine is determined. Moreover, three experimental schemes are designed to compare and analyse the differences between the conventional tangent arrangement model based on IDZs and the intersecting arrangement model based on IDZs.

4.2. Stope Structure Determination. According to the intersecting arrangement model based on IDZs and combined with the stope characteristics of the Yanqianshan Iron Mine, the sublevel height is $22.5 \mathrm{~m}$. The next step is to calculate the optimal drift spacing of elevations from $-213 \mathrm{~m}$ to $-303 \mathrm{~m}$.

The ore-rock bulk flow parameters are determined by ore drawing experiments, with reference to stochastic medium draw theory of Ren. To improve the consistency between the experiment and the mining site, the ore from the stope is used as the experimental material, and the similarity ratio is set to $1: 100$. After three experiments, the experimental results are averaged, and the ore-rock bulk flow parameters of the $-213 \mathrm{~m}$ to $-303 \mathrm{~m}$ sublevels of the Yanqianshan Iron Mine are as follows: $\alpha=1.21, \beta=1.63, \alpha_{1}=1.32$, and $\beta_{1}=1.98$. Based on the mine design, the sublevel height $H$ is $22.5 \mathrm{~m}$, and $k=0.1$.

Because $\alpha=1.21$, the IDZ of the Yanqianshan Iron Mine is similar to an ellipsoid with a thin upper part and thick lower part; according to equation (18), the range of the drift spacing is [17.9106 $\mathrm{m}, 22.4647 \mathrm{~m}$ ].

Using the calculation formula of $V_{o}$ and $V_{r}$, the values of $\alpha, \beta, \alpha_{1}, \beta_{1}, k$ and $\mathrm{H}$ are input, and then $V_{o}$ and $V_{r}$ corresponding to different drift spacings are obtained. Data analysis is performed on $V_{o}$ and $B$ as well as $V_{r}$ and $B$, and then the functional relationship between $V_{o}$ and $B$ as well as that between $V_{r}$ and $B$ are fit (see Figure 7).

$$
\begin{aligned}
& V_{o}=-15.08 \times B^{2}+467.37 \times B-1108.36, \\
& V_{r}=13.90 \times B^{2}-791.98 \times B+9087.28
\end{aligned}
$$

The economic and technical indicators obtained from statistical analysis of the production and economic situation in 2019 by the Technical Department of the Yanqianshan Iron Mine are shown in Table 1.

According to equation (24), the profit generated by recovering one ton of crestal residual ore is $51.09 \mathrm{USD} / \mathrm{t}$; $p=51.09 \mathrm{USD} / \mathrm{t}$.

According to equation (30), the loss generated by mixing one ton of waste rock is $24.2 \mathrm{USD} / \mathrm{t} ; l=24.2 \mathrm{USD} / \mathrm{t}$. 


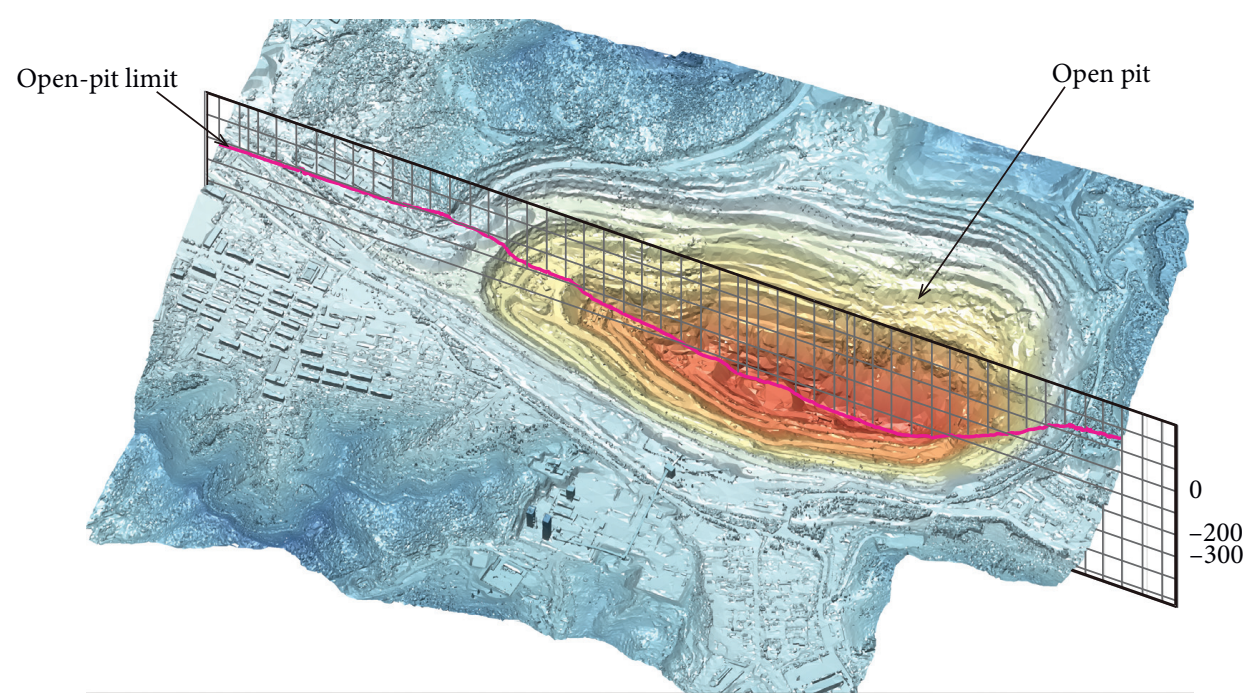

Figure 5: The mining status model of the Yanqianshan Iron Mine.

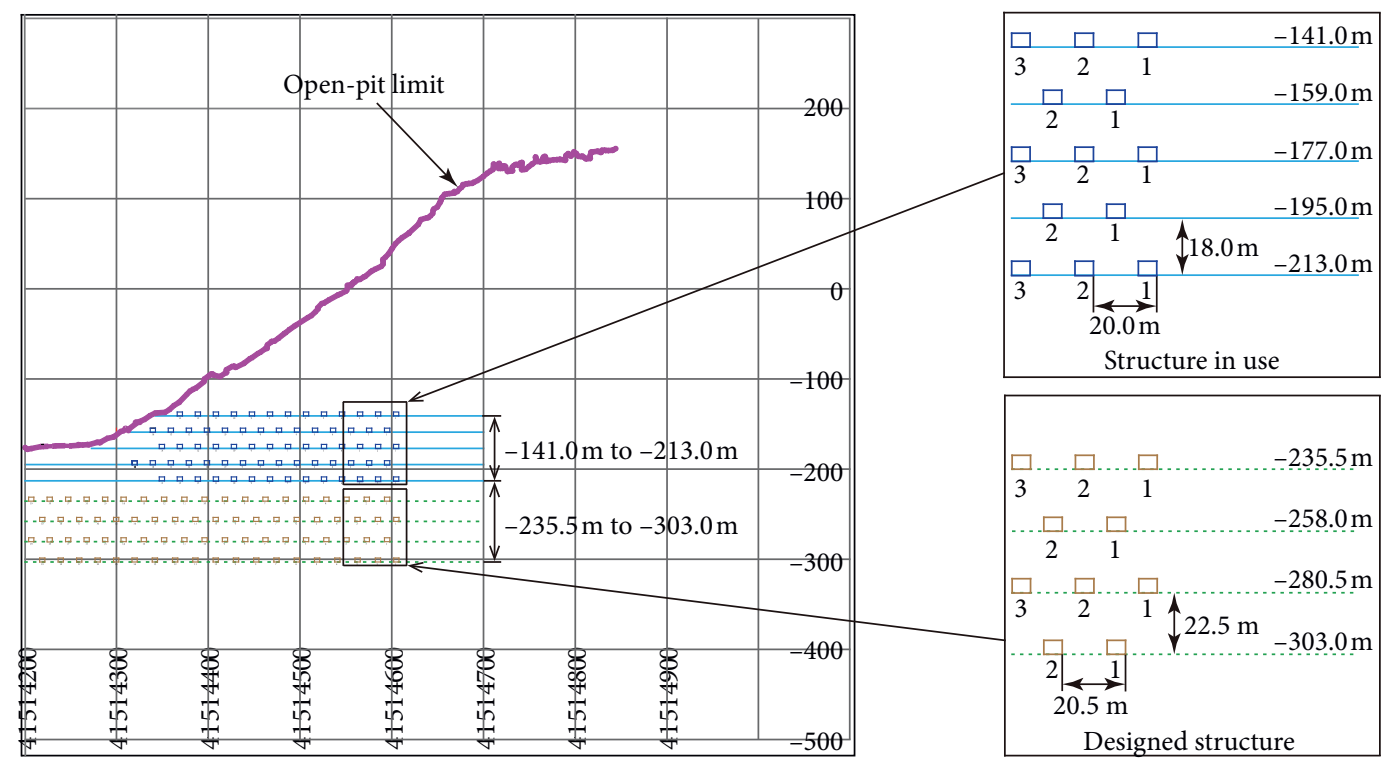

Figure 6: Underground mining structure of the Yanqianshan Iron Mine.

The amount of crestal residual ore recovered is $2 V_{o}$, and the amount of waste rock is $4 V_{r}$ (see Figure 3 ). The ore density is measured as $5.0 \mathrm{t} / \mathrm{m}^{3}$, and the rock density is $3.5 \mathrm{t} /$ $\mathrm{m}^{3}$. According to equations (32)-(34),

$$
s=-12413.47 \times B^{2}+507102.13 \times B-3645031.58 .
$$

According to equation (35), the derivative with respect to $B$ yields $B=20.43 \mathrm{~m}$. Considering the construction and operation of the mine, the optimal approach spacing is set to $20.5 \mathrm{~m}$.

4.3. Physical Simulation Experiments. Based on the above analysis, the stope structure from $-213 \mathrm{~m}$ to $-303 \mathrm{~m}$ in the Yanqianshan Iron Mine is determined, the sublevel height is $22.5 \mathrm{~m}$, and the drift spacing is $20.5 \mathrm{~m}$. To verify the rationality of the scheme, physical simulation experiments are carried out to compare and analyse the loss and dilution degree of ore under the three conditions of the stope structure: the intersecting model, tangent model, and original stope structure.

4.3.1. Materials and Device. Magnetite and dolomite are used as the simulation materials in the experiments, in which magnetite is regarded as the caved ore and dolomite is regarded as the overlying waste rock. The lumpiness ratio of magnetite to stope caved ore is $1: 100$, which is obtained by screening at the crushing station, and the magnetite size is $1 \mathrm{~mm}$ to $3 \mathrm{~mm}$. Dolomite has a slightly larger size than magnetite, ranging from $2 \mathrm{~mm}$ to $3 \mathrm{~mm}$. Due to the fluidity of the ore-rock bulk, the size of overlying waste rock is larger 


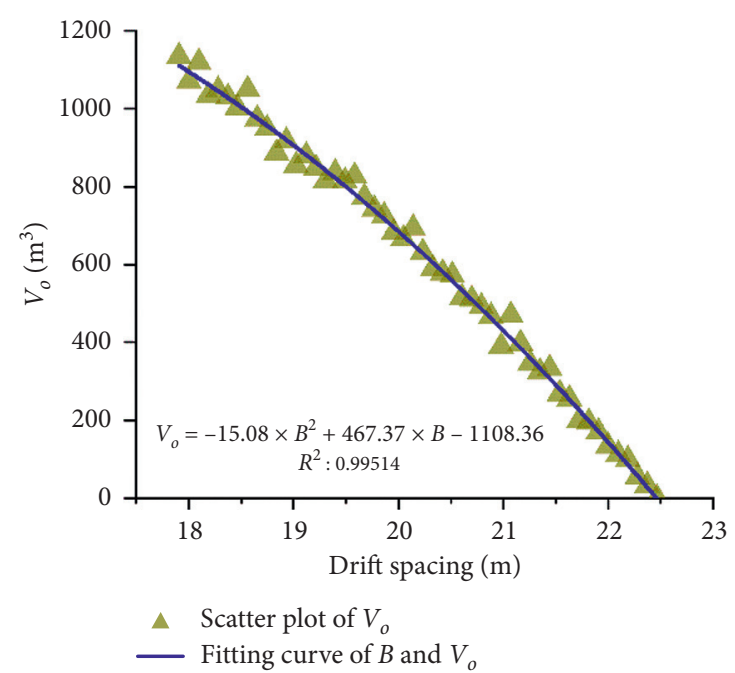

(a)

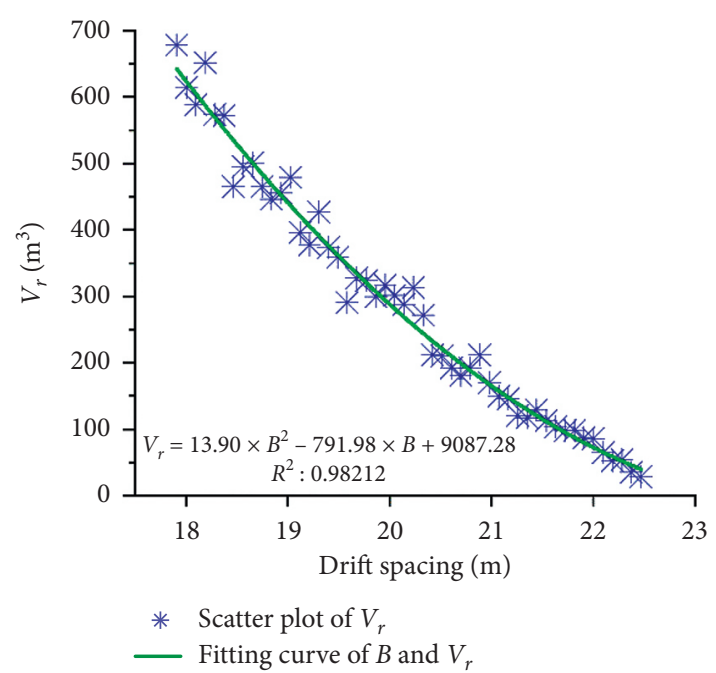

(b)

FIgURE 7: Fitting of the drift spacing and volume. (a) $B$ and $V_{O}$. (b) $B$ and $V_{r}$.

TABle 1: Economic and technical indicators of the Yanqianshan Iron Mine in 2019.

\begin{tabular}{lcc}
\hline Economic and technical indicators & Symbols \& units & Value \\
\hline Ore grade & Co $\%$ & 34 \\
Concentrate grade & $C c \%$ & 65.5 \\
Tailings grade & $C t \%$ & 10 \\
Concentrate price & $P \mathrm{USD} / \mathrm{t}$ & 125.7 \\
Cost loss & $p_{2} \mathrm{USD} / \mathrm{t}$ & 2.96 \\
Cost of waste rock & $l 2 \mathrm{USD} / \mathrm{t}$ & 2.96 \\
\hline
\end{tabular}

than that of caved ore, which can reduce the mixing of waste rock and reduce ore dilution.

A self-designed physical stope model (see Figure 8) with a geometric scale of $1: 100$ is used to compare the loss and dilution degree of ore under the three stope structure conditions. A $10 \mathrm{~mm}$ thick transparent organic glass plate is used to simulate the stope work-plane. The work-plane has five sublevels, each sublevel has 4 or 5 drawpoints, and the drawpoints are in a staggered arrangement shaped like a rhombus. In this experiment, three organic glass plates are made to simulate three groups of different stope structures. An aluminum frame is used to hold the organic glass plates in place to form the stope model. The left, right, and back sides of the stope model are covered with a $10 \mathrm{~mm}$ thick transparent organic glass plate, which is convenient for loading experimental materials and observing the experimental process. There are slots on the left and right sides of the organic glass plate at a distance of $2 \mathrm{~cm}$ from the front stope work-plane to simulate caving space.

4.3.2. Schemes. As presented in Table 2, three physical simulation schemes are designed and 9 groups of experiments are conducted to compare and analyse the loss and dilution degree of the ore under the three stope structure conditions. The objective is to verify the rationality and superiority of the intersecting arrangement model based on IDZs.

4.3.3. Process. The experiment is divided into three steps: filling, drawing, and weighing. During filling, a steel plate should be inserted into the slots of the organic glass plates on the left and right sides to form the caving space, magnetite should be used to fill the caving space, and dolomite should be used to fill the outside of the caving space. Magnetite and dolomite are added simultaneously up to sublevel 5; then, the steel plate is drawn out, and dolomite is continually added to a filling height greater than 2 times the height of a sublevel. The cut-off grade drawing method of the Yanqianshan Iron Mine is simulated for drawing, and the cut-off grade is $20 \%$. To facilitate the experiment operation, the cut-off grade is converted into the waste rock mixing rate, which is calculated to be $26 \%$. For each drawing, the magnetite and dolomite weights are determined, and when the weight of the dolomite divided by the weight of the magnetite and dolomite reaches $26 \%$, drawing at this drawpoint is stopped. Drawing is carried out from top to bottom and from left to right in turn. In the process, the overlying dolomite should be added in a timely manner to maintain a steady drawing pressure. After drawing, the magnetite and dolomite are separated to prepare for the next experiment.

In the drawing process, with the drawing of the ore, the overlying waste rock flows downward, resulting in ore and rock mixtures. When the ore and waste rock are mixed to the cut-off grade, drawing at this drawpoint is stopped. Through the transparent organic glass plate, part of the crestal residual ore can be seen (see Figure 9(a)). When the overlying waste rock is exfoliated layer by layer, residual ore can be seen (see Figure 9(b)). As ore drawing continues, the residual ore moves down with the overlying waste rock and is concentrated in the lowest sublevel. 


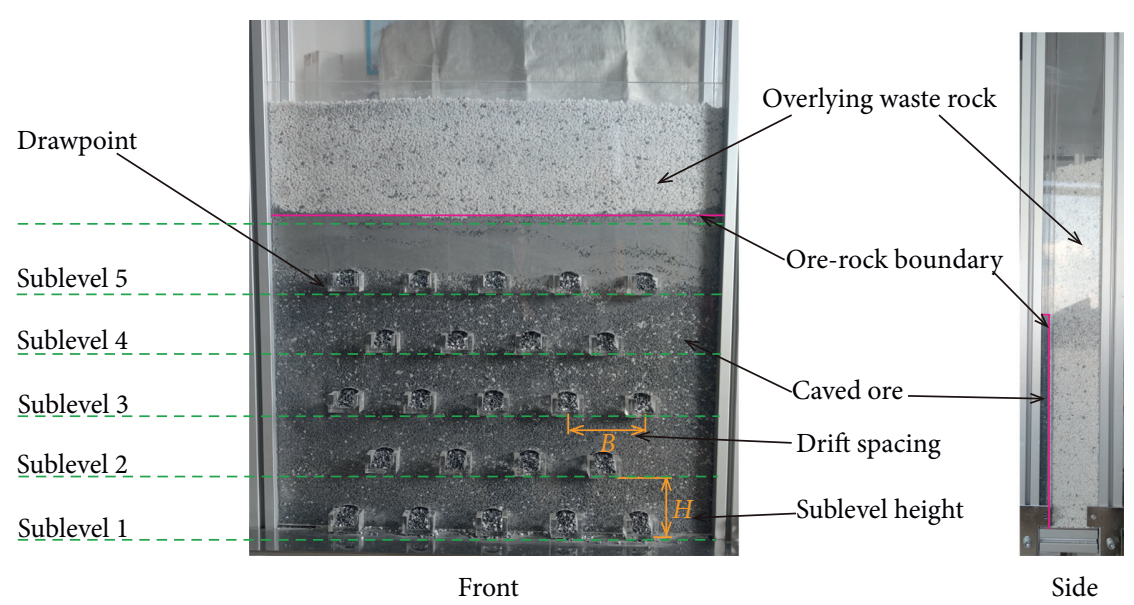

FIgURE 8: Physical stope model of ore drawing.

TABle 2: Experimental schemes.

\begin{tabular}{lccc}
\hline Number & Name & Sublevel height $(\mathrm{m})$ & Drift spacing $(\mathrm{m})$ \\
\hline 1 & Original & 18 & 20 \\
2 & Intersecting model & 22.5 & 20.5 \\
3 & Tangent model & 22.5 & 22.5 \\
\hline
\end{tabular}
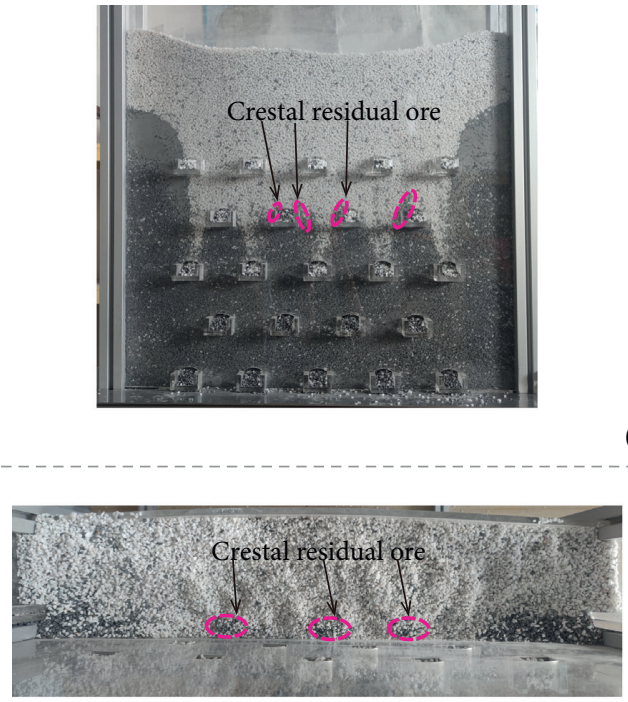

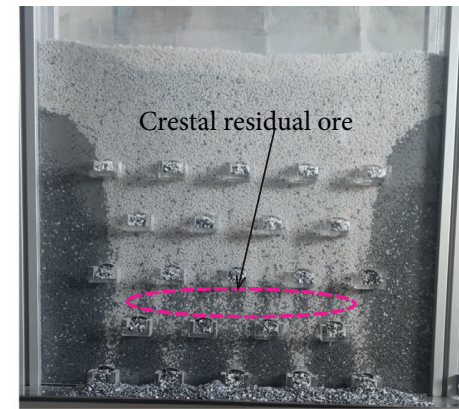

(a)

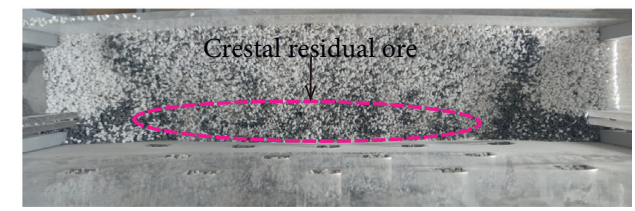

(b)

Figure 9: Drawing phenomenon and effect. (a) Front view. (b) Top view.
4.3.4. Experimental Results and Analysis. Through comparative experiments under different IDZ arrangement models, the ore loss rate and the ore dilution rate are obtained. A comparison of the drawing effects under different IDZ arrangement models is presented in Table 3 and Figure 10.

Table 3 shows that, compared to the original stope structure, a sublevel height of $22.5 \mathrm{~m}$ can effectively reduce the ore loss and dilution rate, which proves the feasibility of large stope structure. When the sublevel height is $22.5 \mathrm{~m}$, the drift spacing is set as $22.5 \mathrm{~m}$ in the tangent model, and the ore loss rate and ore dilution rate are $14.18 \%$ and $12.08 \%$, respectively. However, when the drift spacing is set as $20.5 \mathrm{~m}$ in the intersecting model, the ore loss rate and ore dilution rate are $10.52 \%$ and $12.30 \%$, respectively, and the loss rate is significantly reduced, but the dilution rate changes slightly. Therefore, the intersecting arrangement model based on IDZs can have a beneficial drawing effect. It is further verified that the intersecting arrangement model based on IDZs can reduce the crestal residual ore and improve the recovery of the ore. Although there is also mixing of waste rock, it has little effect on the ore dilution. 
TABLE 3: Comparison of the drawing effects with different IDZ arrangement models.

\begin{tabular}{lcccc}
\hline Number & Stope height $(\mathrm{m})$ & Drift spacing $(\mathrm{m})$ & Loss rate $(\%)$ & Dilution rate $(\%)$ \\
\hline 1 & 18 & 20 & 17.96 & 20.45 \\
2 & 22.5 & 20.5 & 10.52 & 12.30 \\
3 & 22.5 & 22.5 & 14.18 & 12.08 \\
\hline
\end{tabular}

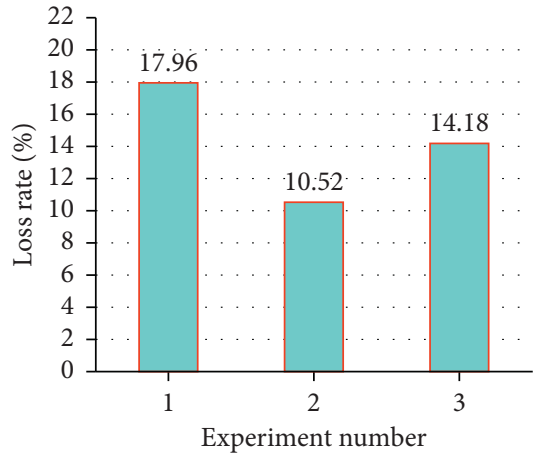

Loss rate (\%)

(a)

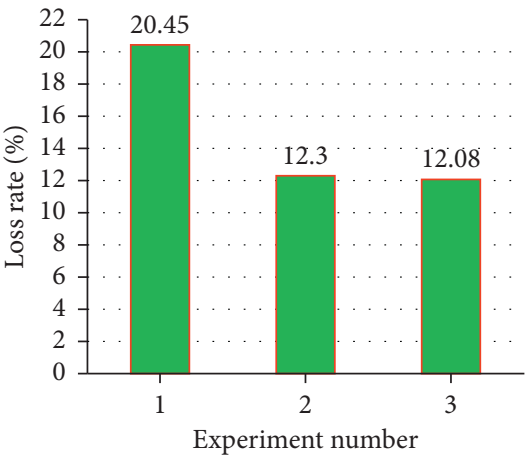

Dilution rate (\%)

(b)

FIgURE 10: A comparison of the drawing effects with different IDZ arrangement models. (a) Loss rate. (b) Dilution rate.

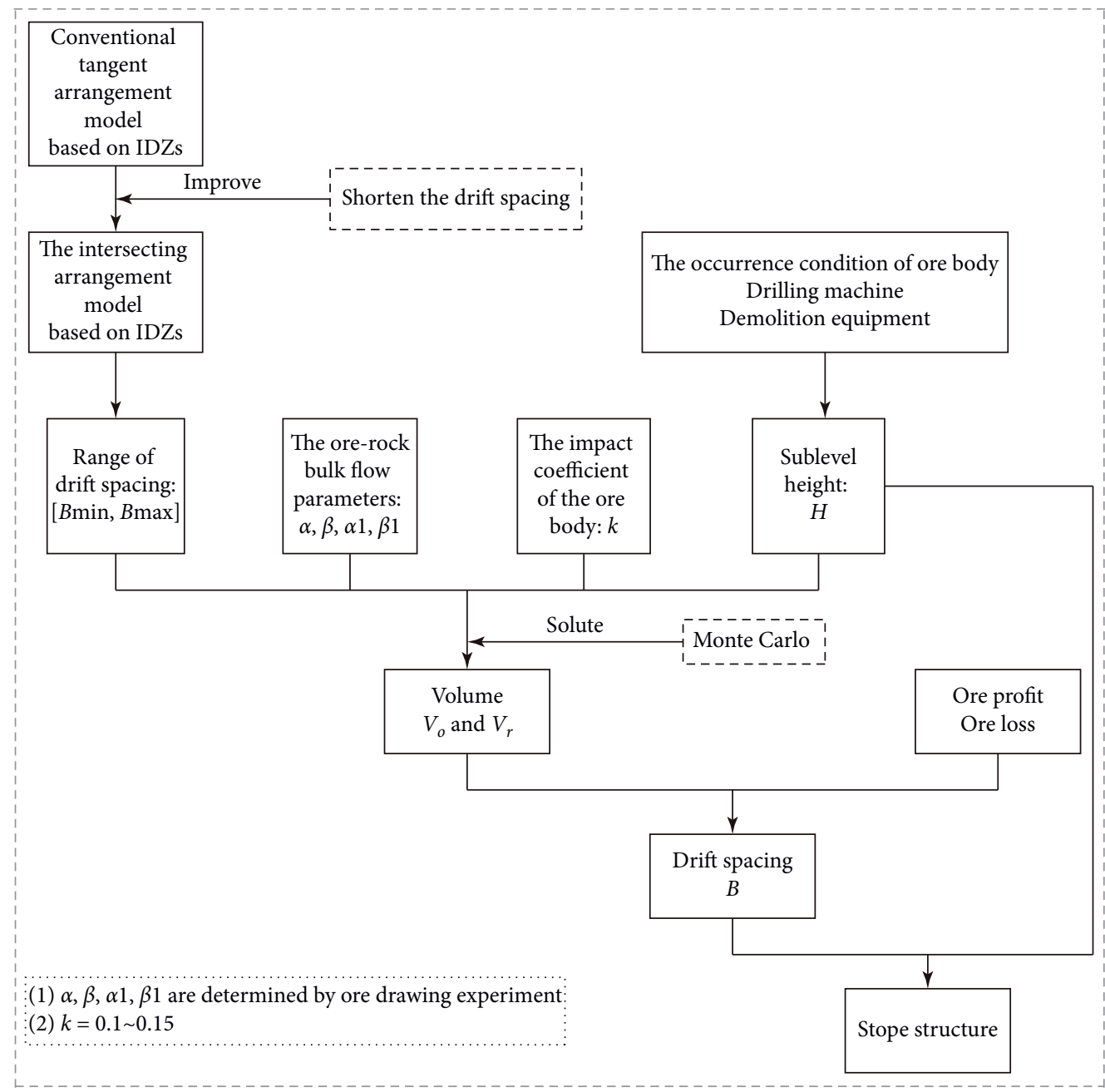

FIGURE 11: Schematic of the dynamic intersecting arrangement model based on IDZs for stope structure optimization. 


\section{Discussion and Conclusion}

The concept of the intersecting arrangement of IDZs was first proposed by Kvapil [3]; later, Laubscher [31] developed and established interaction theory. However, these studies were based on the ellipsoid draw theory, and no reasonable evidence has been provided to quantify the intersecting arrangement of IDZs [32]. In this paper, based on stochastic medium draw theory, a dynamic intersecting arrangement model based on IDZs was established for the case in which the shape of the IDZ is a nonstandard ellipsoid. The dynamic intersecting arrangement model can dynamically adjust the stope structure according to the ore-rock bulk flow parameters, economic indicators, occurrence conditions of the ore body, drilling machine, and so forth. Then, taking the ore profit and loss as the research object, a calculation model was established to quantify the degree of intersection of the IDZs and to determine the best stope structure. The schematic of the study setup is as follows (see Figure 11).

From the analysis of this model (see Figure 11), the main results are as follows:

(1) A dynamic intersecting arrangement model based on IDZs is established

(2) The range of the drift spacing $\left[B_{\min }, B_{\max }\right]$ is determined

(3) With the Monte Carlo algorithm, the calculation method of the ore recovery volume $\left(V_{o}\right)$ and rock mixed volume $\left(V_{r}\right)$ when the IDZs intersect is provided

(4) A calculation model is established for the ore profit and loss

Ore profit: $p=\left(\left(c_{o}-c_{t}\right) /\left(c_{c}-c_{t}\right)\right) \times P-p_{2}$

Ore loss: $l=\left(c_{w} / c_{j} \times\left(1-c_{w}\right)\right) \times P+l_{2}$

(5) A method for determining the drift spacing: $s=V_{O} \times \rho_{O} \times p-V_{r} \times \rho_{r} \times l=f_{1}(B) \times \rho_{o} \times p-$ $f_{2}(B) \times \rho_{r} \times l$ is established, and the drift spacing is determined by finding the derivative with respect to $B$

According to the intersecting arrangement model based on IDZs, the stope structure of elevations from $-213 \mathrm{~m}$ to $-303 \mathrm{~m}$ in the Yanqianshan Iron Mine in China was determined; the optimal drift spacing is $20.5 \mathrm{~m}$, and the sublevel height is $22.5 \mathrm{~m}$. Nine physical experiments under three stope structure conditions, namely, the intersecting model, tangent model, and original stope structure, were carried out. The experimental results showed that the sublevel height of $22.5 \mathrm{~m}$ can effectively reduce the ore loss and dilution rate; at a drift spacing of $20.5 \mathrm{~m}$, the ore loss rate decreased by $3.66 \%$, but the dilution rate changed little, with an increment of $0.22 \%$. This finding verifies the rationality and superiority of the intersecting arrangement model based on IDZs to optimize the stope structure.

An intersecting arrangement model based on Kvapil and Laubscher's interaction theory was proposed in this paper. This model is suitable for the case in which the shape of the IDZ is a nonstandard ellipsoid, and the model is more in line with actual mining conditions. The accurate shape of the IDZ is the basis for stope structure optimization, and future research should focus on the measurement of the shape of the IDZ. Moreover, the model does consider the mechanical properties of the movement of ore-rock bulk when dynamically adjusting the arrangement of IDZs. In future research, mechanical factors should be added to develop a stope structure optimization software program that dynamically adjusts the intersecting arrangement to better guide mine production.

\section{Data Availability}

The data used to support the findings of this study are included within the article.

\section{Conflicts of Interest}

The authors declare that they have no conflicts of interest.

\section{Acknowledgments}

This work was financially supported by the National Natural Science Foundation of China (no. 51534003) and the National Key Research and Development Program of China (no. 2016YFC0801601).

\section{References}

[1] R. Castro and M. Pineda, "The role of gravity flow in the design and planning of large sublevel stopes," Journal of the Southern African Institute of Mining and Metallurgy, vol. 115, no. 2, pp. 113-118, 2015.

[2] Z.-X. Zhang and M. Wimmer, "A case study of dividing a single blast into two parts in sublevel caving," International Journal of Rock Mechanics and Mining Sciences, vol. 104, pp. 84-93, 2018.

[3] I. Janelid and R. Kvapil, "Sublevel caving," International Journal of Rock Mechanics and Mining Sciences \& Geomechanics Abstracts, vol. 3, no. 2, pp. 129-132, 1966.

[4] G. Tao, M. Lu, X. Zhang, R. Zhang, and Z. Zhu, "A new diversion drawing technique for controlling ore loss and dilution during longitudinal sublevel caving," International Journal of Rock Mechanics and Mining Sciences, vol. 113, pp. 163-171, 2019.

[5] A. Jin, H. Sun, S. Wu, and Y. Gao, "Confirmation of the upside-down drop shape theory in gravity flow and development of a new empirical equation to calculate the shape," International Journal of Rock Mechanics and Mining Sciences, vol. 92, pp. 91-98, 2017.

[6] A. Gustafson, H. Schunnesson, J. Paraszczak, G. Shekhar, and P. Brnnman, "Operator influence on the loading process at LKAB's iron ore mines," Journal of the Southern African Institute of Mining and Metallurgy, vol. 120, no. 3, pp. 191-202, 2020.

[7] I. D. Brunton, S. J. Fraser, J. H. Hodgkinson, and P. C. Stewart, "Parameters influencing full scale sublevel caving material recovery at the Ridgeway gold mine," International Journal of Rock Mechanics and Mining Sciences, vol. 47, no. 4, pp. 647-656, 2010.

[8] X. Liu, Theoretical Basis of Ore Drawing, Metallurgical Industry Press, Beijing, China, 1995. 
[9] G. Marano, "The interaction between adjacent draw points in free flowing materials and it application to mining," Chamber of Mines Journal, vol. 22, pp. 25-32, 1980.

[10] H. Ding, S. Chen, S. Chang, G. Li, and L. Zhou, "Prediction of surface subsidence extension due to underground caving: a case study of Hemushan iron mine in China," Mathematical Problems in Engineering, vol. 2020, Article ID 5086049, 10 pages, 2020.

[11] M. ГM, Drawing of Caving Ore Block, Metallurgical Industry Press, Beijing, China, 1958.

[12] F. Ren, The Stochastic Medium Draw Theory and its Application, Qiyun Qing, Beijing, China, 1994.

[13] J. Litwiniszyn, "Application of the equation of stochastic processes to mechanics of loose bodies," Archives of Mechanics, vol. 8, no. 4, pp. 393-411, 1956.

[14] R. Kvapil, Underground Mining Methods Handbook, Society of Mining, Metallurgy and Explorations, Englewood, Colorado, USA, 1982.

[15] K. Yu, F. Ren, G. Chitombo, R. Puscasu, and L. Kang, "Optimum sublevel height and drift spacing in sublevel cave mining based on random medium theory," Mining, Metallurgy \& Exploration, vol. 37, no. 2, pp. 681-690, 2020.

[16] P. Yuan, J. Zhao, Y. Ju, and S. Gong, "Study on large-space structural parameters of pillarless sublevel caving," Metal Mine, vol. 6, pp. 5-8, 2004.

[17] L. Wang, A. Shao, X. Liu, L. Yang, and H. Ding, "New computational framework for modeling the gravity flow behavior of sublevel caving material," Computers and Geotechnics, vol. 125, Article ID 103675, 2020.

[18] B. Tan, Z. Zhang, R. He, and Q. Zhu, "Discussion on the rationality and experimental research of the ore-drawing ellipsoid arrangement theory," Journal of Northeastern University (Natural Science), vol. 40, no. 7, pp. 1014-1019, 2019.

[19] F. Melo, F. Vivanco, C. Fuentes, and V. Apablaza, "On drawbody shapes: from Bergmark-Roos to kinematic models," International Journal of Rock Mechanics and Mining Sciences, vol. 44, no. 1, pp. 77-86, 2007.

[20] R. J. McCormick, "How wide does a drawpoint draw?" Engineering and Mining Journal, pp. 106-166, 1968.

[21] R. Castro, R. Trueman, and A. Halim, "A study of isolated draw zones in block caving mines by means of a large $3 \mathrm{D}$ physical model," International Journal of Rock Mechanics and Mining Sciences, vol. 44, no. 6, pp. 860-870, 2007.

[22] Q. Chen, F. Zhao, Q. Chen, Y. Wang, Y. Zhong, and W. Niu, "Orthogonal simulation experiment for flow characteristics of ore in ore drawing and influencing factors in a single funnel under a flexible isolation layer," JOM-Journal of the Minerals, vol. 69, no. 12, pp. 2485-2491, 2017.

[23] X. Liu, G. Zhang, and X. Liu, "Analysis of ore loss and dilution in sublevel caving," Metal Mine, vol. 1, pp. 53-60, 2006.

[24] S. Liu, J. Zhang, and B. Zhu, "Volume computation using a direct Monte Carlo method," Computing \& Combinatorics, vol. 4598, pp. 198-209, 2007.

[25] L. M. Popescu, "A geometry modeling system for ray tracing or particle transport Monte Carlo simulation," Computer Physics Communications, vol. 150, no. 1, pp. 21-30, 2003.

[26] E. J. Elbrond, "Economic effects of ore losses and rock dilution," CIM Bulletin, vol. 87, pp. 131-134, 1994.

[27] Z. Zhang, X. Liu, and G. Yu, Sublevel Caving Method without Sill Pillars in the Base of Undiluted Ore Draw: Undiluted Ore Draw Theory and its Practice in Mine, Northeastern University Press, Shenyang, China, 2007.

[28] J. Ming, L. Zhang, and J. Sun, “Analysis models of technical and economic data of mining enterprises based on big data analysis," in Proceedings of the 2018 the 3rd IEEE International Conference on Cloud Computing and Big Data Analysis, pp. 224-227, Chengdu, China, April 2018.

[29] A. L. Shao and H. L. Feng, "The prediction of northern slope deformation and failure caused by the transformation of open-pit to underground mining in the Yanqianshan iron mine," Applied Mechanics and Materials, vol. 580-583, pp. 364-370, 2014.

[30] Y. Hu, F. Ren, H. Ding, Y. Fu, and B. Tan, "Study on the process and mechanism of slope failure induced by mining under open pit slope: a case study from Yanqianshan iron mine, China," Advances in Civil Engineering, vol. 2019, Article ID 6862936, 26 pages, 2019.

[31] D. H. Laubscher, "Cave mining-the state of the art," The Journal of the South African Institute of Mining and Metallurgy, vol. 94, no. 10, pp. 279-293, 1994.

[32] A. A. E. Halim, Study of the Influence of Interactive of Interactive Draw upon Drawpoint Spacing in Block and Sublevel Caving Mines, University of Queensland, Brisbane, Australia, 2006. 